\title{
Adventitial ablation technique that permits the assessment of adventitial-dependent contribution to microvascular contractile function
}

\author{
Satirah Zainalabidin, Roger M. Wadsworth, Paul Coats \\ Strathclyde Institute of Pharmacy and Biomedical Sciences, University of Strathclyde, \\ Glasgow G4 0RE, Scotland, UK
}

This is the peer-reviewed, accepted author manuscript of the following publication: Zainalabidin, S.,

Wadsworth, R. M., \& Coats, P. (2016). Adventitial ablation technique that permits the assessment of adventitialdependent contribution to microvascular contractile function. Analytical Biochemistry, 499, 71-77.

10.1016/j.ab.2016.01.009 


\begin{abstract}
:
Resistance arteries have been implicated as major contributing factor in the sequela of disease conditions such as hypertension and diabetes and as such are a major focus of cardiovascular research. The paracrine influence of the intimal endothelial layer of resistance arteries is well established. Considering the growing body of evidence substantiating a functionally relevant vascular adventitia in this present study we have established a technique which permits determination of the functional influence of the adventitial layer on resistance artery tone. Isolating adventitial-dependent function, analogous to isolating endothelial function, has potentially significant implication for studying the as yet unexplored role of the microvascular adventitial layer in modulating acute vascular contractile function.
\end{abstract}

Key words: Microcirculation; Adventitia; Vasoconstriction; Vasorelaxation; Pressure Autoregulation 


\section{Introduction}

Blood vessels comprise of three anatomically and physiologically distinct layers; the intimal endothelium; the medial vascular smooth muscle and the outer adventitial layer. To date vascular research has focused largely on the endothelium and smooth muscle dependent function and the interplay between both layers in the regulation of vascular tone in health and disease [1]. Historically the adventitial layer has been considered as a passive encapsulating structure containing perivascular sympathetic nerves and in large blood vessels a vasa vasorum. However, an increasing number of studies have demonstrated a distinct physiological role for the adventitia [2]. Studies have demonstrated the adventitia in large calibre arteries play a part in atherogenesis and more recently as a modulator of agonistdependent responses [3,4]. Moreover, the adventitial layer has been proposed to be potentially crucial in vascular remodelling phenomena and has intriguingly been reported as the "first responder" during early vascular disease development [4]. Many studies have documented structural adaptation of the medial vascular smooth muscle layer in disease conditions however the adventitial layer similarly undergoes structural adaptation [1,5]. Human subcutaneous and skeletal muscle resistance arteries underwent hypotrophic remodelling with the greatest structural loss observed within the adventitial layer [6]. Similar to large calibre arteries it is feasible that adventitial-derived signalling molecules such as reactive oxygen species may modulating underlying vascular smooth muscle contractile function [2]. Excluding trans-nerve stimulation studies there are no other studies focusing on specific adventitial derived cell signalling intermediaries in small resistance arteries. Considering the importance of small resistance artery function in health and disease and the growing body of evidence pointing towards a likely significant role of the adventitial layer as an important contributor to both acute (contractile) and chronic (structural) vascular function in larger calibre arteries the development of a method for isolating vascular adventitialdependent function analogous to endothelial removal in small resistance arteries would permit the advancement of focused adventitial-dependent microvascular research. Thus the aim of this study was to establish a reliable and reproducible method of functionally isolating adventitial-dependent function in small resistance arteries.

\section{Methods}

\subsection{Isolation of rat middle cerebral arteries}

All procedures were performed under the UK Animals (Scientific Procedures) Act 1986 and were approved by the Ethical Review Committee of the University of Strathclyde. Adult male Sprague-Dawley rats (12 weeks, 250 300g) were killed by cervical dislocation. The brain was removed and the middle cerebral arteries (MCA; outer diameter $170 \pm 2.8 \mu \mathrm{m}$ ) were dissected from surrounding connective tissue under a dissection microscope (WPI, UK; Model SSZ 10x/22) and placed in ice-cold physiological saline solution (PSS). 


\subsection{Adventitial removal following collagenase digest}

Following isolation both ends of resistance arteries (5mm lengths) were closed by tying sutures around the ends to protect the luminal compartment. Arteries were placed in eppendorf tubes filled with Krebs solution containing collagenase type II, in a $37^{\circ} \mathrm{C}$ shaking water bath [3]. The protocol was repeated with incubation times of 5, 7.5, 10 and 15 mins and collagenase concentrations of $1,1.5$ and $2 \mathrm{mg} \mathrm{ml}^{-1}$. Following collagenase exposure arteries were immediately rinsed with ice cold PSS. The arteries were then pinned at both ends to a sylgard-based dissecting dish and the adventitia was carefully removed by gentle stripping with Dumont \#5 fine forceps (WPI, UK) under a dissecting microscope.

\subsection{Adventitial ablation by paraformaldehyde exposure}

As above both ends of the resistance artery were tied off. The adventitial layer was ablated by dipping the artery in $4 \%$ formaldehyde buffered saline (FBS) for 5 seconds and thereafter immediately rinsed in ice cold PSS. In this study arteries undergoing treatment to remove or ablate the adventitial layer are referred to as ADV- (experimental) and intact adventitial arteries (control) as ADV+. As a control measure ADV+ arteries were exposed to an identical processing method as ADV-, the only difference being ADV+ were dipped in PSS as opposed to $4 \%$ FBS.

\subsection{Functional confirmation of resistance artery function}

Successful removal/ ablation of the adventitia were immediately measured by trans-nerve stimulation (TNS) of adventitial perivascular nerve-dependent modulation of vascular tone. Disruption of adventitial-dependent function was confirmed by loss of TNS-dependent contraction $(1 \sim 16 \mathrm{~Hz} / 50-130 \mathrm{v})$ and loss of TNS/ nicotine-dependent relaxation (in the presence of guanethidine).

\subsection{Resistance artery function: wire myography and transmural nerve stimulation (TNS)}

Arterioles were bathed in PSS and gassed with $20 \% \mathrm{O}_{2} / 5 \% \mathrm{CO}_{2} / 75 \%$ nitrogen maintaining $\mathrm{pH} 7.4$ at $37^{\circ} \mathrm{C}$. MCA were mounted on a Danish MyoTech 4 channel myograph model 410P (Aarhus, Denmark) using the standard technique [7]. Recording of isometric tension/ force generated by the arteries were measured using an ADI PowerLab 4/25 bridge amplifier/digitizer (Chalgrove, UK) and intercept Chart Software. Two electrodes were placed on either side of wire myograph mounted arteries. TNS was applied using a S44D Grass stimulator (Grass technologies, Slough, UK) with the following parameters 40-130 V, $0.3-0.5 \mathrm{msec}$ pulse duration, increasing frequency $(1,2,4,8 \& 16 \mathrm{~Hz})$. The neurogenic nature of the TNS response was confirmed following 30 minute incubation with the postganglionic sympathetic nerve terminal blocker guanethidine $(10 \mu \mathrm{M})$.

\subsection{Resistance artery function: pressure myography}

Isolated arterioles were studied using previously described techniques (Coats et al, 2003). Briefly, arterioles were mounted on a Danish MyoTech (Aarhus, Denmark) P110 pressure myograph system and secured with two $17 \mu \mathrm{M}$ nylon sutures to size-matched micro-cannulae. 
Pressure-independent responses to contractile agonists were measured at an intraluminal pressure of $40 \mathrm{mmHg}$. Pressure-dependent myogenic responses were studied by increasing intraluminal pressure from $40-120 \mathrm{mmHg}$ in $40 \mathrm{mmHg}$ steps for a period of 5 minutes at each pressure step. Myogenic tone (\%) was calculated using the formula (LD in $\mathrm{Ca}^{2+}$ free PSS $\mathrm{LD}$ in $\mathrm{Ca}^{2+} \mathrm{PSS} / \mathrm{LD} \mathrm{Ca}{ }^{2+}$ free $\mathrm{x} 100$. Where $\mathrm{LD} \mathrm{Ca}^{2+}$ is the lumen diameter in the presence of $\mathrm{Ca}^{2+}$ and $\mathrm{LD} \mathrm{Ca}^{2+}$ free the lumen diameter in the absence of $\mathrm{Ca}^{2+}$.

\subsection{Chemiluminescence measurements of ROS}

ROS produced from MCAs was measured by Superoxide production by control and collared carotid artery ring segments was measured using $5 \mu \mathrm{mol} / \mathrm{L}$ lucigenin-enhanced chemiluminescence. Vessel segments (dry weight $10 \pm 0.5 \mathrm{ug}$ ) were pre-incubated in PSS at $37^{\circ} \mathrm{C}$ with $\mathrm{NADH}^{+}$(substrate for Nox, $100 \mu \mathrm{M}$ ) and diethyldithiocarbamate (DETCA, 10 $\mathrm{mM}$ ) a superoxide dismutase inhibitor for $30 \mathrm{~min}$. Arteries were transferred into KrebsHEPES buffer containing ANG II $(0.1 \mu \mathrm{M}), \mathrm{NADH}^{+}(100 \mu \mathrm{M})$ and lucigenin in plastic cuvettes. Luminescence was measured in relative light unit per second (RLU/s) every 10 seconds over a period of 200 seconds using a Sirius L luminometer (Sirius, Germany).

\subsection{Assessment of vascular mechanics}

Circumferential stress $(\sigma)$, force generated per unit area of the arteriole wall thickness (WT) as a product of intraluminal pressure (IP), was calculated using the formula:

$$
\sigma=\left(\Delta \operatorname{IPr}_{\mathrm{i}}\right) /(2 \lambda \mathrm{WT})
$$

$\Delta \mathrm{P}$ being the transmural pressure difference and $\mathrm{r}_{\mathrm{i}}$ being the luminal radius at a given IP. Circumferential strain $(\varepsilon)$, fractional changes in lumen diameter (LD) as a product of pressure, was calculated from arteriole lumen diameter measurements using the formula:

$$
\varepsilon=(\mathrm{LD}-\mathrm{Do}) / \mathrm{Do}
$$

where Do is the original diameter, $5 \mathrm{mmHg}$ was used as the original diameter in the calculation of $\varepsilon$. To determine the passive stiffness (resistance to pressure-dependent stretch) of the artery under study the stress-strain data for individual arteries were fitted to an exponential curve $\left(y=a e^{b x}\right)$ to obtain the tangential elastic modulus:

$$
\mathrm{E}=\sigma_{\text {orig }} \mathrm{e}^{\beta \varepsilon}
$$

Where oorig is the stress at the original diameter $(5 \mathrm{mmHg})$ and $\beta$ is the slope of the tangential elastic modulus.

2.9. Vascular tissue viability and reactive oxygen species measurement using confocal microscopy

Tissue viability: Successful adventitial ablation and confirmation of underlying vascular smooth muscle and endothelial viability/ integrity following paraformaldehyde dipping were established by laser-scanning confocal microscopy (Leica SP-5; Leica DM6000) and x20 (N.A. 0.75) air objective and x40 (N.A. 1.3) oil objective on pressurised (40mmHg) vessels 
[6]. Immediately following the ablation procedure viable or non-viable cells/ layers of tissue were identified using an inclusion/ exclusion criteria using the flurophores propidium iodide (non-permeable in viable cells; Sigma-Aldrich, Poole, UK) $10 \mu \mathrm{g} \mathrm{ml}^{-1}$ in PSS; excitation/ emission 495/640-nm) and Hoescht 33342 (Sigma-Aldrich, Poole, UK, 100nmol 1-1 excitation/ emission 350/461-nm). Confocal images were processed using Volocity software (Lexington, MA).

Reactive oxygen species measurement: Intact segments of artery were incubated with $10 \mu \mathrm{mol} \mathrm{l}^{-1}$ 5-(and-6)-chloromethyl-dichlorodihydrofluorescein diacetate, acetyl ester (DCFDA, Invitrogen) for $30 \mathrm{~min}$ and ROS was observed by confocal microscopy in response to stimulation with angiotensin II (ANG II). The vascular smooth muscle layer were measured in the z-axis using the x60 water immersion objective (Nikon N.A. 1.3). A z-plane stack of images were collected where the step interval was set at $1 \mu \mathrm{M}$. The medial smooth muscle layer was determined by the first brightest to last brightest nuclei. The mean fluorescent intensity from each z-stack image was measured and these values averaged to give a mean fluorescent intensity for the whole smooth muscle layer using ImageJ.

\subsection{Drugs and reagents}

Physiological saline solution (PSS): composition (mmol l-1) - $\mathrm{NaCl} 119, \mathrm{KCl} 4.5, \mathrm{NaHCO}_{3}$ 25, $\mathrm{KH}_{2} \mathrm{PO}_{4}$ 1.0, $\mathrm{MgSO}_{4}$ 1.2, glucose 10 and $\mathrm{CaCl}_{2}$ 2.5. $\mathrm{Ca}^{2+}$ free PSS composition was identical to that of PSS other than the removal of $\mathrm{Ca}^{2+}$ and addition of $1 \mathrm{mM}$ EGTA. KrebsHEPES composition (mmol l-1) - $\mathrm{NaCl} 118, \mathrm{KCl} 4.5, \mathrm{MgSO}_{4} 1.2, \mathrm{CaCl}_{2} 2.5, \mathrm{KH}_{2} \mathrm{PO}_{4} 1.2$, $\mathrm{NaHCO}_{3} 25$, HEPES 10, glucose 10. All drugs and reagents were purchased from Sigma (Poole, Dorset, UK) and all were prepared on the day of the experiment. Collagenase PSS:

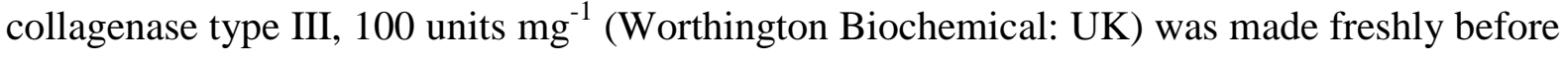
each experiment and diluted with PSS.

\subsection{Data and statistical analysis}

Values are presented as mean \pm standard error of the mean (SEM). Contraction data to vasoconstrictor agonists are represented as percentage contraction relative to the maximum contraction evoked by $60 \mathrm{mmol} \mathrm{l}^{-1} \mathrm{~K}^{+} \mathrm{PSS}$. Relaxation data are represented as percentage relaxation relative to the pre-constricted diameter of the artery. Statistical analysis of the data was performed using Graph Pad Prism (USA). Comparisons between groups were made using paired or unpaired Student's t test or ANOVA for repeated measures. Significance was assumed if $\mathrm{p}<0.05$.

\section{Results}

\subsection{Adventitial removal following collagenase exposure}

Following the 15 minute collagenase $\left(1.5 \mathrm{mg} \mathrm{ml}^{-1}\right)$ exposure protocol (Gonzalez et al 2001) functional integrity was measured. Collagenase exposure alone (with no stripping) under the published experimental conditions resulted in complete loss of fundamental contractile function. Reducing the concentration of collagenase to $0.5 \mathrm{mg} / \mathrm{ml}$ and reducing the time of 
exposure from 15 minutes to 5 minutes failed to improve contractile function (Fig. 1A). Visual analysis of resistance arteries by confocal microscopy revealed propidium iodide positive staining across all layers of the vessels following collagenase exposure (Fig. 1B). In keeping with the original published study collagenase protocols were tested in the larger calibre rat aorta tissue [3]. In aorta collagenase treatment alone or collagenase treatment plus careful stripping of the adventitial layer had no deleterious effect on vascular contractile function nor did it result in any propidium iodide positive staining of the vascular smooth muscle or endothelial layers (data not presented).

\subsection{Adventitial ablation using $4 \%$ formaldehyde buffered saline}

Following 5 second dipping resistance artery function was measured. In contrast to the collagenase exposure protocol FBS exposure had no detrimental effect on receptorindependent contractile function (Fig. 1C). Vascular cellular integrity was confirmed by confocal microscopy. Fig. 1D shows no propidium iodide positive medial or intimal cells following FBS exposure. As expected the outer adventitial layer shows positive staining (Fig. 1D).

\subsection{Functional confirmation of adventitial ablation}

TNS was used to measure neurogenic-dependent contraction. Fig. 2A shows a TNSdependent increase in vascular contractile tone which was significantly reduced following incubation with guanethidine $(10 \mu \mathrm{M})$. The effect of adventitial ablation with transient dipping in 4\% FBS almost eliminated the TNS-dependent response. Additional confirmation of adventitial ablation was investigated using nicotine $(10 \mu \mathrm{M})$ in the presence of guanethidine. Fig. 2B shows significant loss of nicotine-dependent relaxation in ADV-vs. $\mathrm{ADV}+(54.1 \pm 3.1 \%$ vs. $12.8 \pm 3.1 \%$ respectively; $\mathrm{p}<0.05)$. Adventitial fibroblasts have been shown to liberate significant amounts of reactive oxygen species (ROS) [7]. Fig. 2C shows the effect of adventitial ablation on angiotensin II $(0.1 \mathrm{uM})$ induced ROS. Following treatment the capacity of MCAs to liberate ROS as measured by chemiluminescence was significantly reduced to $\sim 50 \%$ in ADV- MCAs (Fig 2C.).

\subsection{Pressure-independent tone}

In MCAs at intraluminal pressure of $40 \mathrm{mmHg}$ and following transient exposure to $4 \% \mathrm{FBS}$ we observed no loss of underlying vascular smooth muscle receptor-independent (Fig. 3A) or receptor-dependent tone (Figs. 3B, 3C). Nor were endothelium-independent (Fig. 3D) or endothelium-dependent relaxation (Fig. 3E) affected.

\subsection{Pressure-dependent tone}

Despite having no effect on pressure-independent mechanism of vascular tone transient dipping in 4\% FBS significantly reduced the well documented pressure-dependent function in MCAs. Fig. $3 \mathrm{G}$ shows pressure-diameter relationship in $\mathrm{ADV}+, \mathrm{ADV}+\mathrm{in}^{2+}$ free $\mathrm{PSS}$ and ADV-. In the presence of $\mathrm{Ca}^{2+}$ and in response to increasing pressure from $40-80-120 \mathrm{mmHg}$ mean vessel diameter reduced (175-165-163 $\mu \mathrm{m}$ respectively). In paraformaldehyde dipped 
vessels loss of pressure-dependent myogenic tone (Fig. 3G) was greatest at $120 \mathrm{mmHg}$ $(\mathrm{ADV}+\mathrm{vs}$. ADV-; $24.8 \pm 2.2 \%$ vs. $9.4 \pm 3.3 \%$ respectively; $\mathrm{p}<0.05)$.

\subsection{Pressure-dependent passive mechanical properties following FBS exposure}

Transient dipping in 4\% FBS may have resulted in partial fixation. Therefore we measured the effect of $4 \%$ formaldehyde exposure on pressure-dependent deformation in an attempt to identify if the loss of pressure-dependent tone in ADV- was due to partial fixation of the outer layer of the artery. However, Fig. 3H highlights there was in fact no degree of partial fixation as measured by the passive pressure-dependent mechanical properties of the arteries. Fig. $3 \mathrm{H}$ exemplifies these observations showing no significant difference in the stress-strain relationship ADV+ vs. ADV-. Moreover no significant difference was observed in the tangent of the elastic modulus values $(\beta)$ in ADV+ vs. ADV-. $(\beta-10.4 \pm 2.4$ vs. $10.1 \pm$ 1.3 respectively).

\subsection{Vascular smooth muscle reactive oxygen species measurement}

Figure 4 shows mean fluorescent activity within the vascular wall in response to angiotensin II $(1 \& 10 \mathrm{uM})$. Of note there is no significant difference between ROS measured in the muscle layer pre and post transient dipping in 4\% FBS. The no loss of function as a consequence of transient dipping in 4\% FBS is further confirmed as we clearly observe similar angiotensin II dose-dependent increase in ROS in both ADV+ and ADV-.

\section{Discussion}

Previously adventitial-dependent functional isolation has been achieved in larger conduit arteries using an enzymatic digest technique [3]. We have confirmed this technique in the present study using rat aorta however we have importantly demonstrated this technique is not suitable for smaller calibre resistance artery studies. In this study we have shown for the first time a technique for isolating adventitial-dependent functional processes in small resistance arteries. We are confident that the method for adventitial ablation only affects adventitial dependent processes. Enzymatic processing resulted in complete loss of tonic mechanisms. The deleterious effect of the enzymatic exposure was quantified by confocal microscopy where the viability of all cells across the vascular wall stained positive for PI. In complete contrast transient dipping in 4\% FBS followed by PSS rinsing produced only PI positive staining of the outer adventitial layer. Functionally the loss of TNS and nicotine dependent tone combined with the functional preservation of the underlying smooth muscle and endothelium function confirms specificity of this method. Additional confirmation of the specificity of functional adventitial ablation using this technique was gained by the loss of ROS as measured by chemiluminescence. Similar to large arteries the adventitial layer of resistance arteries are populated with many fibroblast cells with a large potential to produce ROS [6]. The data presented in Figure $2 \mathrm{C}$ shows a significantly reduced total ROS liberated from the ADV- artery when compared with the ADV+ artery. Whereas figure 4 highlights the amount of ROS measured within the muscle layer of the artery remains unchanged 
suggesting that the reduced ROS measured in the ADV- (Figure 2C) is a consequence of transient 4\% FBS dipping and loss of adventitial function. Thus this technique of isolating adventitial function in small resistance arteries is one potential focus of future research of the role of adventitial fibroblast-derived ROS and vascular reactivity.

One unexpected observation was the effect of adventitial ablation on pressuredependent myogenic tone. To the best of our knowledge this is the first observation showing a potential role of the adventitia layer in modulating acute pressure-dependent myogenic tone. Mechanisms underlying myogenic tone have yet to be fully elucidated. However ROS has been implicated as a co-signalling molecule underlying the myogenic response and adventitial-derived ROS in addition to ROS from the underlying smooth muscle could conceivably be involved in modulating the actin cytoskeleton [8]. Moreover ROS is known to modulate $\mathrm{Ca}^{2+}$ signalling and potassium channel activation $[9,10]$. There is clear lack of consensus regarding the location and nature of the initiating bio-sensor underlying myogenic tone; it is not unconceivable that the location of the sensory apparatus may be situated within the adventitial-medial interface [11]. Clearly this observation requires further investigation however goes some way to exemplifying the potential important application of this technique.

Although the data has not presented within this manuscript similar observations were measured in third order mesenteric arteries thus the implications of this work for resistance artery studies are potentially important. Parallels can be drawn when considering the vascular endothelium in health and disease where the early use of endothelial removal studies to identify endothelial-dependent function were commonly adopted. Following this present study we are now in a position to undertake similar work to further elucidate the role of the adventitial layer in modulating small resistance artery function.

\section{References}

[1] M.J. Mulvany, Modeling the vasculature: a judicious approach? Hypertension 46 (2005) 652-3.

[2] F.A. Auger, P. D'Orléans, L. Germain, Adventitia contribution to vascular contraction: Hints provided by tissue-engineered substitutes Cardiovas Res 75 (2007) 669-678

[3] M.C. González, S.M. Arribas, F. Molero, M.S. Fernández-Alfonso, Effect of removal of adventitia on vascular smooth muscle contraction and relaxation Am J Physiol 280 (2001) 2876-2881.

[4] M.J. Haurani, P.J. Pagano, Adventitial fibroblast reactive oxygen species as autocrine and paracrine mediators of remodeling: Bellwether for vascular disease? Cardiovasc Res 75 (2007) 679-689. 
[5] S. Sartore, A. Chiavegato, E. Faggin, R. Franch, M. Puato, S. Ausoni, Contribution of adventitial fibroblasts to neointima formation and vascular remodeling: from innocent bystander to active participant. Circ Res 89 (2001) 1111-21.

[6] P. Coats, Y.P. Jarajapu, C. Hillier, J.C McGrath, C. Daly, The use of fluorescent nuclear dyes and laser scanning confocal microscopy to study the cellular aspects of arterial remodelling in human subjects with critical limb ischaemia. Exp Physiol 88 (2003) 547-54.

[7] M.J. Mulvany, W Halpern, Contractile properties of small arterial resistance vessels in spontaneously hypertensive and normotensive rats. Circ Res 41 (1977) 19-26.

[8] P.T. Nowicki, S. Flavahan, H. Hassanain, S. Mitra, S. Holland, P.J. GoldschmidtClermont, N.A. Flavahan, Redox signaling of the arteriolar myogenic response. Circ Res 89 (2001) 114-6.

[9] K. Tokube, T. Kiyosue, M. Arita, Effects of hydroxyl radicals on KATP channels in guineapig ventricular myocytes. Pflügers Arch 437 (1998)155-157.

[10] K.M. Lounsbury, Q. Hu, R.C. Ziegelstein, Calcium signaling and oxidant stress in the vasculature. Free Radic Biol Med 28 (2000) 1362-9.

[11] D.R. Harder, J. Narayanan, D. Gebremedhin, Pressure-induced myogenic tone and role of 20-HETE in mediating autoregulation of cerebral blood flow. Am J Physiol 300 (2011) H1557-65.

\section{Figure legends}

Figure 1. The effect of collagenase-dependent adventitial stripping on microvascular function. A. Contractile response to $60 \mathrm{mM} \mathrm{K}{ }^{+} \mathrm{PSS}$ in $\mathrm{ADV}+$ and following 3 min exposure to collagenase $\left(0.2 \mathrm{mg} \mathrm{ml}^{-1}\right)$ collagenase treated and collagenase treated plus adventitial stripping of mid-cerebral resistance arteries. $\mathrm{n}=4$ pairs; ${ }^{*} \mathrm{p}<0.05$, t-test, treatment vs. ADV+. (B) Confocal image of a mid-cerebral resistance artery following $3 \mathrm{~min}$ exposure to collagenase $(0.2 \mathrm{mg} / \mathrm{ml})$ showing propidium iodide positive red staining in all layers of the artery wall (C) Contractile response of mid-cerebral resistance arteries to $60 \mathrm{mmol}^{-1} \mathrm{~K}^{+} \mathrm{PSS}$ before and after $5 \mathrm{sec}$ dipping in $4 \%$ formalin buffered saline ( $\mathrm{n}=9$ pairs) (D) Confocal image of propidium iodide (PI) stained mid-cerebral resistance artery following $5 \mathrm{sec}$ dipping in $4 \%$ formalin buffered saline. Red nuclei are positive for PI; autofluorescing structures within the artery wall shown by the green. (Scale bar $1 \mathrm{~B}$ and $1 \mathrm{D}=50 \mu \mathrm{m}$ ) 
Figure 2. Adventitial-dependent microvascular function following paraformaldehyde exposure. A, TNS-dependent contraction at $16 \mathrm{~Hz}$ before and after incubation with guanethidine $(10 \mu \mathrm{M})$ in $\mathrm{ADV}+$ arteries and $16 \mathrm{~Hz}$-dependent contraction in ADV-arteries; Contraction data are expressed as a $\%$ of the maximum contraction to $60 \mathrm{mmol}^{-1} \mathrm{~K}^{+} \mathrm{PSS}$. * p<0.05 ADV+ vs. Guanethidine/ ADV+, n=6, t-test; \# p<0.05 ADV- vs. ADV+, n=6, t-test. $\mathrm{B}$, Shows nicotine-dependent relaxation (in the presence of $10 \mu \mathrm{M}$ guanethidine); $* \mathrm{p}<0.05, \mathrm{t}$ test, $\mathrm{ADV}$ - vs. $\mathrm{ADV}+, \mathrm{n}=6 . \mathrm{C}$, Measurement of reactive oxygen species by chemiluminescence in angiotensin II $(0.1 \mu \mathrm{M})$ challenged MCAs. * $\mathrm{p}<0.05, \mathrm{ADV}-\mathrm{vs}$. ADV+, one way ANOVA for repeated measures, $n=6$.

Figure 3. Highlights contractile functional analysis in resistance arteries following transient dipping in 4\% FBS. A, receptor-independent mechanisms of contraction evoked by potassium supplemented PSS (4.5-100 $\mathrm{mmol}^{-1}$; $\left.\mathrm{n}=6\right)$. B and C highlight receptordependent contraction evoked by U46199 ( $n=6)$ and 5-hydroxytryptamine (5HT; n=6) respectively. $\mathrm{D}$ and $\mathrm{E}$ show the effect on endothelium-independent relaxation and endothelium-dependent relaxation evoked by sodium nitroprusside (SNP; n=6) and acetylcholine (ACh; $n=6$ ) respectively. $F$, pressure-diameter relationship in rat mid-cerebral resistance arteries. * $\mathrm{p}<0.05 \mathrm{ADV}$ - vs. $\mathrm{ADV}+(\mathrm{ANOVA}) \mathrm{n}=9$, and pressure-diameter relationship following removal of $\mathrm{Ca}^{2+} \# \mathrm{p}<0.05 \mathrm{ADV}+/ \mathrm{ADV}-\mathrm{vs}$. $\mathrm{Ca}^{2+}$ free $(\mathrm{n}=7)$. G, effect of paraformaldehyde dipping on pressure-dependent myogenic tone, * $\mathrm{p}<0.05$ ADV- vs. $\mathrm{ADV}+$ at 80 and $120 \mathrm{mmHg}$, ANOVA for repeated measures, $\mathrm{n}=9 . \mathrm{H}$, highlights no change in pressure-dependent mechanical properties as measured by the stress-strain relationship in $\mathrm{ADV}+$ and $\mathrm{ADV}$ - arteries, $\mathrm{n}=8$.

Figure 4. Measurement of ROS in the vascular smooth muscle wall of small resistance arteries using laser scanning confocal microscopy. Stimulation with $1 \mathrm{uM}$ angiotensin II (Ang II) produced $\sim 3.5$ fold increase in ROS when compared with background unstimulated control $(\bullet p<0.05,1$ uM Ang II vs. Control, $n=6)$. The liberation of ROS further increased to $\sim 6$ fold above background when stimulated with $10 \mathrm{uM}$ Ang II and was again significantly increased when compared with 1uM Ang II (* $\mathrm{p}<0.05$ 10uM Ang II vs. 1uM Ang II, $n=6)$. In all cases there were no differences measured when comparing ADV- vs. ADV+. 
Figure 1.

A

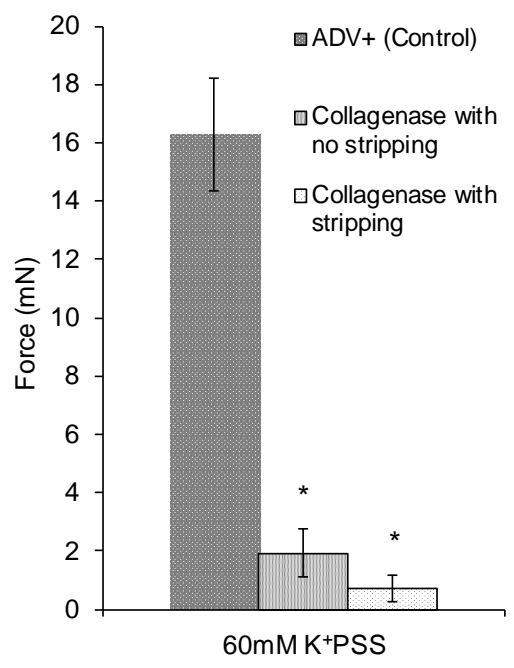

C

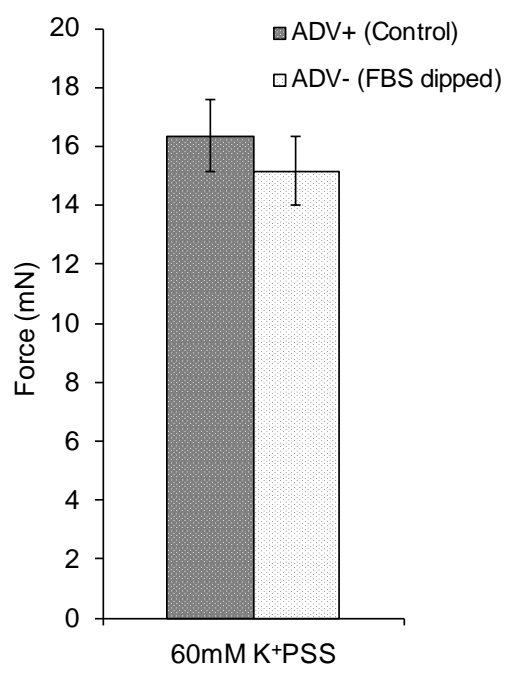

B

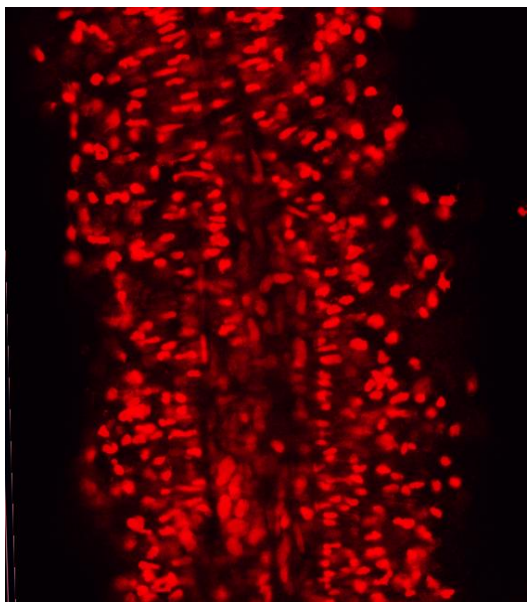

D

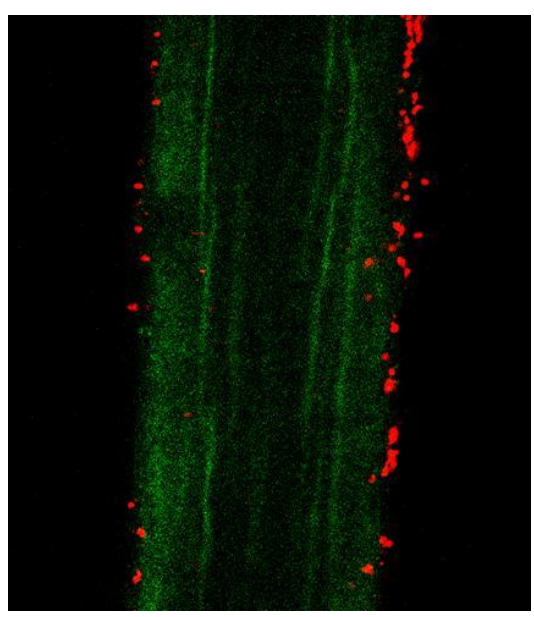


Figure 2.
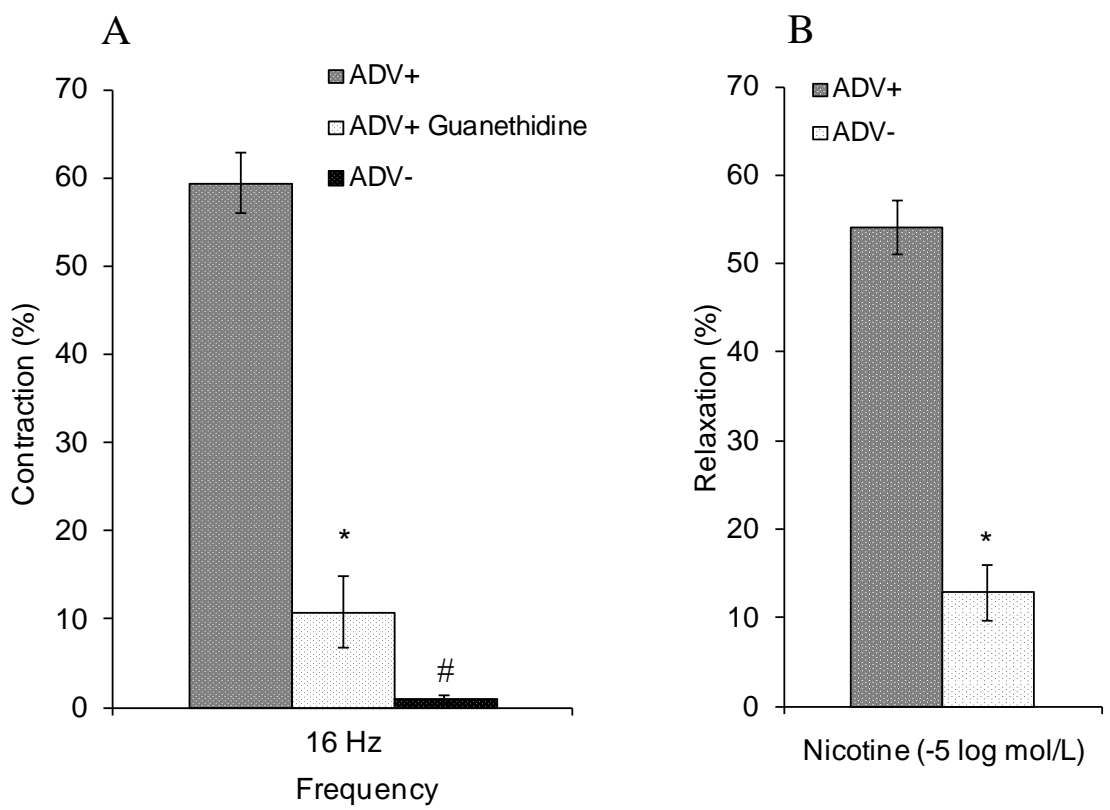

C

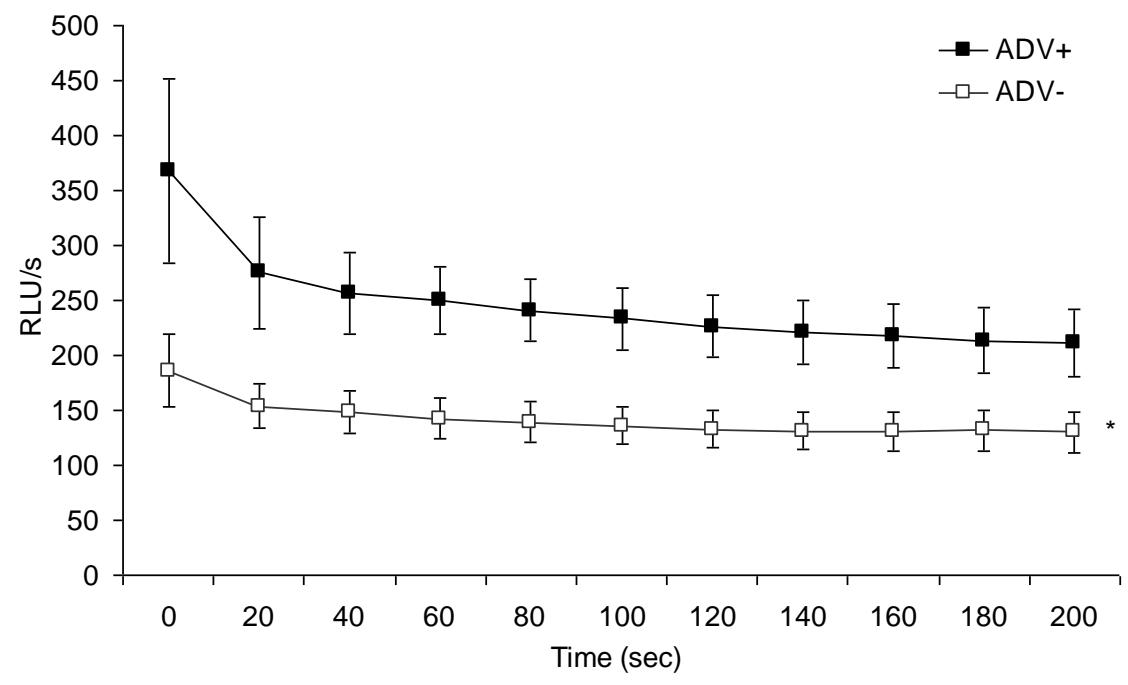


Figure 3.

A

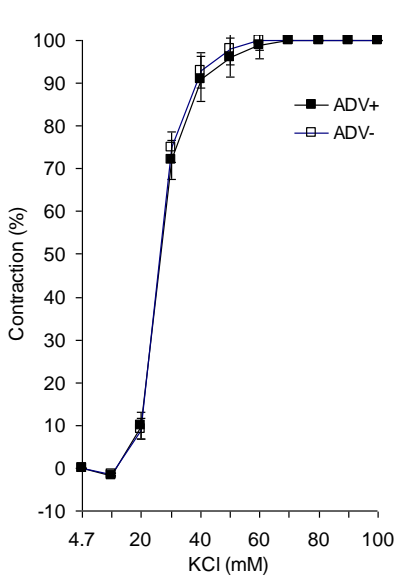

D

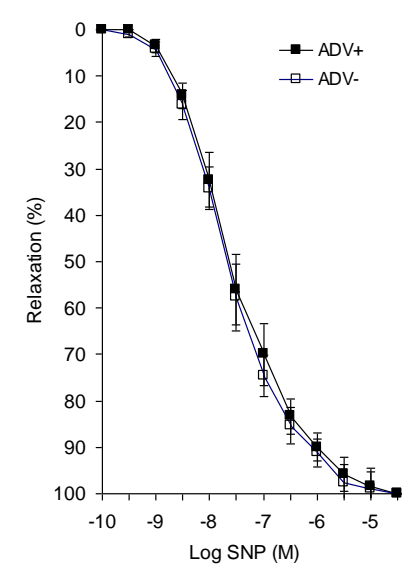

F

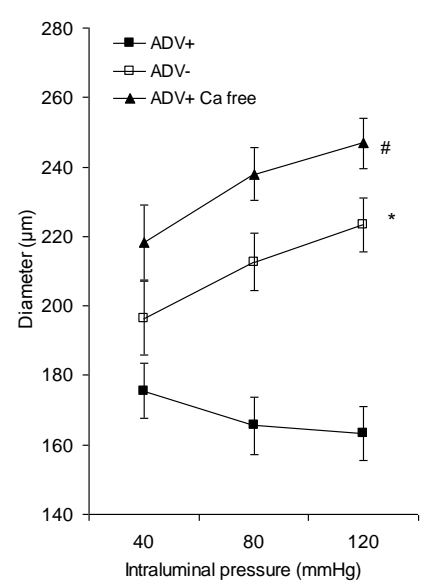

B

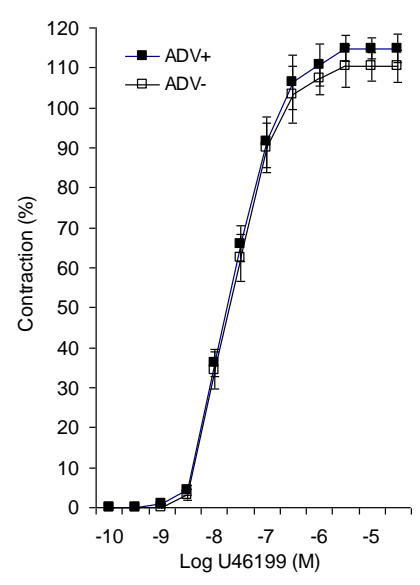

C

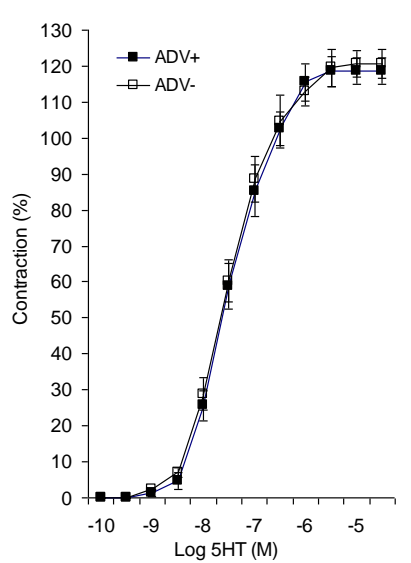

E

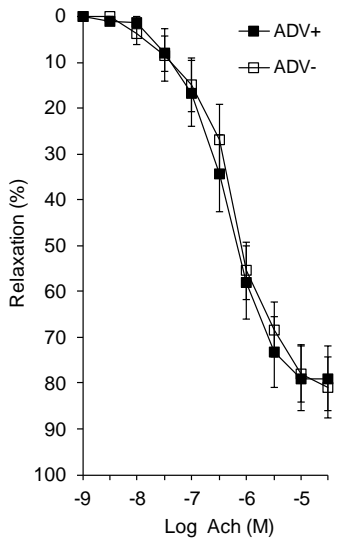

H
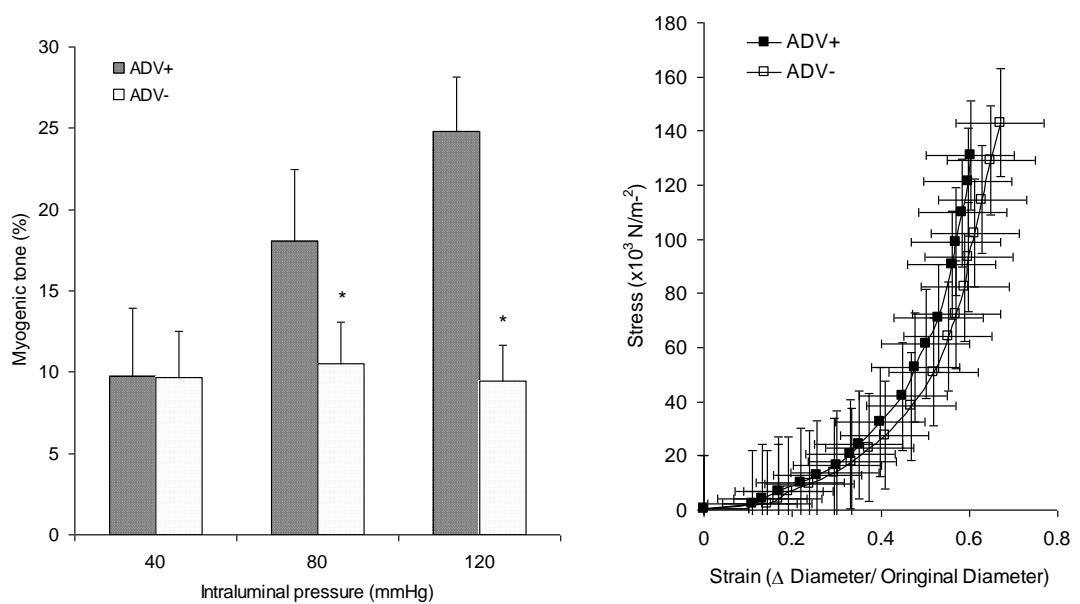

Figure 4

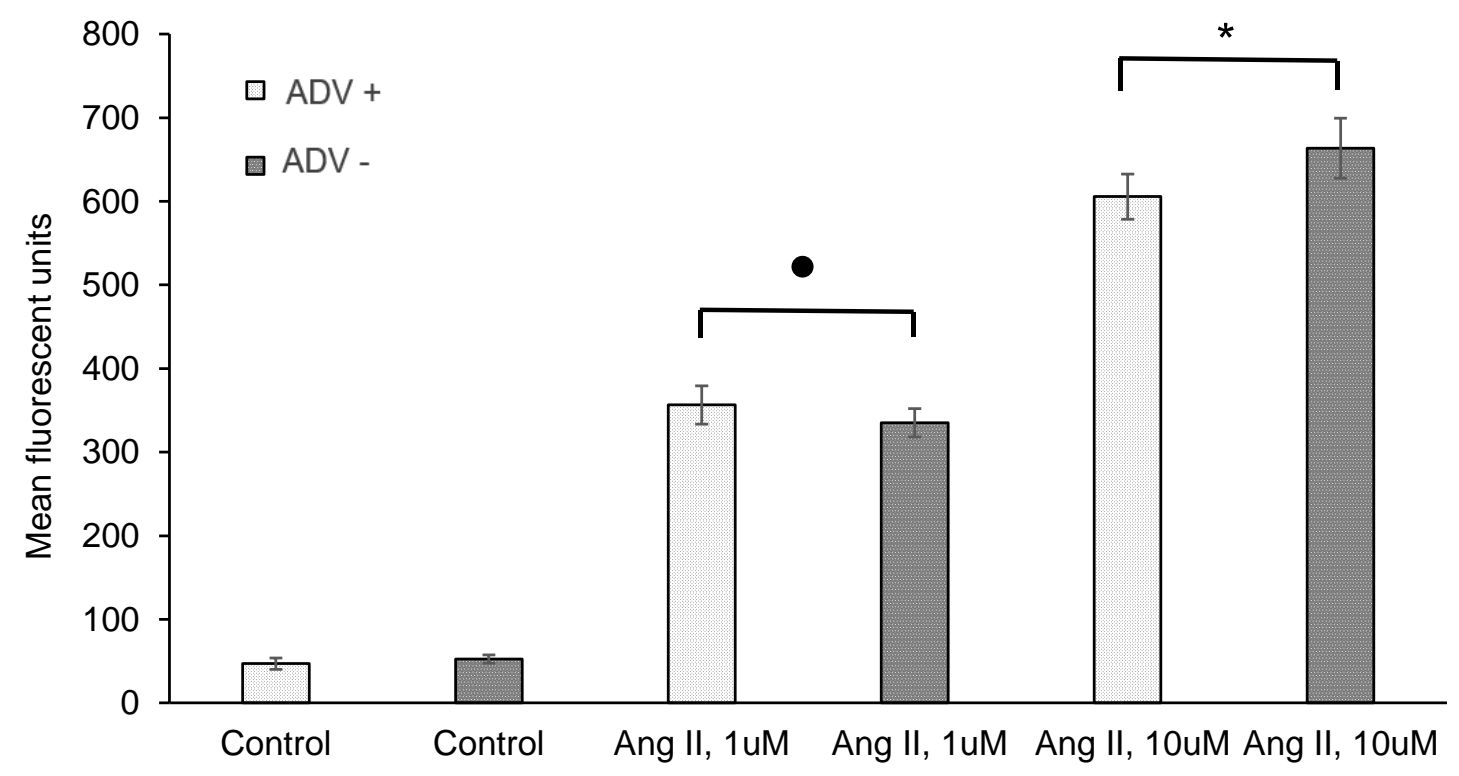


Dear Reviewers

07.01.16

Re: Manuscript- Adventitial ablation permits the assessment of adventitial-dependent contribution to resistance artery tone

Thank-you for your review. Following consideration of comment regarding the repetitive use of "Shows the..." I have amended the legend text as suggested

Kind regards

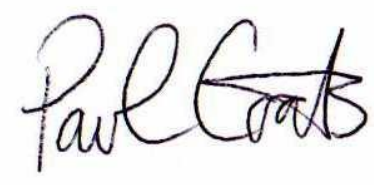

Paul Coats (PhD)

paul.coats@strath.ac.uk

SIPBS

University of Strathclyde

161 Cathedral Street

Glasgow

Scotland

UK

Tel 00441415485790

Fax 00441415522563 
Title: Adventitial ablation technique that permits the assessment of adventitial-dependent contribution to microvascular contractile function

Authors: Zainalabidin S. Coats P.

Short title: Adventitial-dependent microvascular function

Word count: 2532

Strathclyde Institute of Pharmacy \& Biomedical Sciences

University of Strathclyde

161 Cathedral Street

Glasgow

G4 0RE

Scotland

Tel: $0044(0) 1415485790$

Fax: $0044(0) 1415522562$

Email for correspondence; paul.coats@ @strath.ac.uk 


\begin{abstract}
:
Resistance arteries have been implicated as major contributing factor in the sequela of disease conditions such as hypertension and diabetes and as such are a major focus of cardiovascular research. The paracrine influence of the intimal endothelial layer of resistance arteries is well established. Considering the growing body of evidence substantiating a functionally relevant vascular adventitia in this present study we have established a technique which permits determination of the functional influence of the adventitial layer on resistance artery tone. Isolating adventitial-dependent function, analogous to isolating endothelial function, has potentially significant implication for studying the as yet unexplored role of the microvascular adventitial layer in modulating acute vascular contractile function.
\end{abstract}

Key words: Microcirculation; Adventitia; Vasoconstriction; Vasorelaxation; Pressure Autoregulation 


\section{Introduction}

Blood vessels comprise of three anatomically and physiologically distinct layers; the intimal endothelium; the medial vascular smooth muscle and the outer adventitial layer. To date vascular research has focused largely on the endothelium and smooth muscle dependent function and the interplay between both layers in the regulation of vascular tone in health and disease [1]. Historically the adventitial layer has been considered as a passive encapsulating structure containing perivascular sympathetic nerves and in large blood vessels a vasa vasorum. However, an increasing number of studies have demonstrated a distinct physiological role for the adventitia [2]. Studies have demonstrated the adventitia in large calibre arteries play a part in atherogenesis and more recently as a modulator of agonistdependent responses [3,4]. Moreover, the adventitial layer has been proposed to be potentially crucial in vascular remodelling phenomena and has intriguingly been reported as the "first responder" during early vascular disease development [4]. Many studies have documented structural adaptation of the medial vascular smooth muscle layer in disease conditions however the adventitial layer similarly undergoes structural adaptation [1,5]. Human subcutaneous and skeletal muscle resistance arteries underwent hypotrophic remodelling with the greatest structural loss observed within the adventitial layer [6]. Similar to large calibre arteries it is feasible that adventitial-derived signalling molecules such as reactive oxygen species may modulating underlying vascular smooth muscle contractile function [2]. Excluding trans-nerve stimulation studies there are no other studies focusing on specific adventitial derived cell signalling intermediaries in small resistance arteries. Considering the importance of small resistance artery function in health and disease and the growing body of evidence pointing towards a likely significant role of the adventitial layer as an important contributor to both acute (contractile) and chronic (structural) vascular function in larger calibre arteries the development of a method for isolating vascular adventitialdependent function analogous to endothelial removal in small resistance arteries would permit the advancement of focused adventitial-dependent microvascular research. Thus the aim of this study was to establish a reliable and reproducible method of functionally isolating adventitial-dependent function in small resistance arteries.

\section{Methods}

\subsection{Isolation of rat middle cerebral arteries}

All procedures were performed under the UK Animals (Scientific Procedures) Act 1986 and were approved by the Ethical Review Committee of the University of Strathclyde. Adult male Sprague-Dawley rats (12 weeks, 250 300g) were killed by cervical dislocation. The brain was removed and the middle cerebral arteries (MCA; outer diameter $170 \pm 2.8 \mu \mathrm{m}$ ) were dissected from surrounding connective tissue under a dissection microscope (WPI, UK; Model SSZ 10x/22) and placed in ice-cold physiological saline solution (PSS). 


\subsection{Adventitial removal following collagenase digest}

Following isolation both ends of resistance arteries (5mm lengths) were closed by tying sutures around the ends to protect the luminal compartment. Arteries were placed in eppendorf tubes filled with Krebs solution containing collagenase type II, in a $37^{\circ} \mathrm{C}$ shaking water bath [3]. The protocol was repeated with incubation times of 5, 7.5, 10 and 15 mins and collagenase concentrations of $1,1.5$ and $2 \mathrm{mg} \mathrm{ml}^{-1}$. Following collagenase exposure arteries were immediately rinsed with ice cold PSS. The arteries were then pinned at both ends to a sylgard-based dissecting dish and the adventitia was carefully removed by gentle stripping with Dumont \#5 fine forceps (WPI, UK) under a dissecting microscope.

\subsection{Adventitial ablation by paraformaldehyde exposure}

As above both ends of the resistance artery were tied off. The adventitial layer was ablated by dipping the artery in $4 \%$ formaldehyde buffered saline (FBS) for 5 seconds and thereafter immediately rinsed in ice cold PSS. In this study arteries undergoing treatment to remove or ablate the adventitial layer are referred to as ADV- (experimental) and intact adventitial arteries (control) as ADV+. As a control measure ADV+ arteries were exposed to an identical processing method as ADV-, the only difference being ADV+ were dipped in PSS as opposed to $4 \%$ FBS.

\subsection{Functional confirmation of resistance artery function}

Successful removal/ ablation of the adventitia were immediately measured by trans-nerve stimulation (TNS) of adventitial perivascular nerve-dependent modulation of vascular tone. Disruption of adventitial-dependent function was confirmed by loss of TNS-dependent contraction $(1 \sim 16 \mathrm{~Hz} / 50-130 \mathrm{v})$ and loss of TNS/ nicotine-dependent relaxation (in the presence of guanethidine).

\subsection{Resistance artery function: wire myography and transmural nerve stimulation (TNS)}

Arterioles were bathed in PSS and gassed with $20 \% \mathrm{O}_{2} / 5 \% \mathrm{CO}_{2} / 75 \%$ nitrogen maintaining $\mathrm{pH} 7.4$ at $37^{\circ} \mathrm{C}$. MCA were mounted on a Danish MyoTech 4 channel myograph model 410P (Aarhus, Denmark) using the standard technique [7]. Recording of isometric tension/ force generated by the arteries were measured using an ADI PowerLab 4/25 bridge amplifier/digitizer (Chalgrove, UK) and intercept Chart Software. Two electrodes were placed on either side of wire myograph mounted arteries. TNS was applied using a S44D Grass stimulator (Grass technologies, Slough, UK) with the following parameters 40-130 V, $0.3-0.5 \mathrm{msec}$ pulse duration, increasing frequency $(1,2,4,8 \& 16 \mathrm{~Hz})$. The neurogenic nature of the TNS response was confirmed following 30 minute incubation with the postganglionic sympathetic nerve terminal blocker guanethidine $(10 \mu \mathrm{M})$.

\subsection{Resistance artery function: pressure myography}

Isolated arterioles were studied using previously described techniques (Coats et al, 2003). Briefly, arterioles were mounted on a Danish MyoTech (Aarhus, Denmark) P110 pressure myograph system and secured with two $17 \mu \mathrm{M}$ nylon sutures to size-matched micro-cannulae. 
Pressure-independent responses to contractile agonists were measured at an intraluminal pressure of $40 \mathrm{mmHg}$. Pressure-dependent myogenic responses were studied by increasing intraluminal pressure from $40-120 \mathrm{mmHg}$ in $40 \mathrm{mmHg}$ steps for a period of 5 minutes at each pressure step. Myogenic tone (\%) was calculated using the formula (LD in $\mathrm{Ca}^{2+}$ free PSS $\mathrm{LD}$ in $\mathrm{Ca}^{2+} \mathrm{PSS} / \mathrm{LD} \mathrm{Ca}{ }^{2+}$ free $\mathrm{x} 100$. Where $\mathrm{LD} \mathrm{Ca}^{2+}$ is the lumen diameter in the presence of $\mathrm{Ca}^{2+}$ and $\mathrm{LD} \mathrm{Ca}^{2+}$ free the lumen diameter in the absence of $\mathrm{Ca}^{2+}$.

\subsection{Chemiluminescence measurements of ROS}

ROS produced from MCAs was measured by Superoxide production by control and collared carotid artery ring segments was measured using $5 \mu \mathrm{mol} / \mathrm{L}$ lucigenin-enhanced chemiluminescence. Vessel segments (dry weight $10 \pm 0.5 \mathrm{ug}$ ) were pre-incubated in PSS at $37^{\circ} \mathrm{C}$ with $\mathrm{NADH}^{+}$(substrate for Nox, $100 \mu \mathrm{M}$ ) and diethyldithiocarbamate (DETCA, 10 $\mathrm{mM}$ ) a superoxide dismutase inhibitor for $30 \mathrm{~min}$. Arteries were transferred into KrebsHEPES buffer containing ANG II $(0.1 \mu \mathrm{M}), \mathrm{NADH}^{+}(100 \mu \mathrm{M})$ and lucigenin in plastic cuvettes. Luminescence was measured in relative light unit per second (RLU/s) every 10 seconds over a period of 200 seconds using a Sirius L luminometer (Sirius, Germany).

\subsection{Assessment of vascular mechanics}

Circumferential stress $(\sigma)$, force generated per unit area of the arteriole wall thickness (WT) as a product of intraluminal pressure (IP), was calculated using the formula:

$$
\sigma=\left(\Delta \operatorname{IPr}_{\mathrm{i}}\right) /(2 \lambda \mathrm{WT})
$$

$\Delta \mathrm{P}$ being the transmural pressure difference and $\mathrm{r}_{\mathrm{i}}$ being the luminal radius at a given IP. Circumferential strain $(\varepsilon)$, fractional changes in lumen diameter (LD) as a product of pressure, was calculated from arteriole lumen diameter measurements using the formula:

$$
\varepsilon=(\mathrm{LD}-\mathrm{Do}) / \mathrm{Do}
$$

where Do is the original diameter, $5 \mathrm{mmHg}$ was used as the original diameter in the calculation of $\varepsilon$. To determine the passive stiffness (resistance to pressure-dependent stretch) of the artery under study the stress-strain data for individual arteries were fitted to an exponential curve $\left(y=a e^{b x}\right)$ to obtain the tangential elastic modulus:

$$
\mathrm{E}=\sigma_{\text {orig }} \mathrm{e}^{\beta \varepsilon}
$$

Where oorig is the stress at the original diameter $(5 \mathrm{mmHg})$ and $\beta$ is the slope of the tangential elastic modulus.

2.9. Vascular tissue viability and reactive oxygen species measurement using confocal microscopy

Tissue viability: Successful adventitial ablation and confirmation of underlying vascular smooth muscle and endothelial viability/ integrity following paraformaldehyde dipping were established by laser-scanning confocal microscopy (Leica SP-5; Leica DM6000) and x20 (N.A. 0.75) air objective and x40 (N.A. 1.3) oil objective on pressurised (40mmHg) vessels 
[6]. Immediately following the ablation procedure viable or non-viable cells/ layers of tissue were identified using an inclusion/ exclusion criteria using the flurophores propidium iodide (non-permeable in viable cells; Sigma-Aldrich, Poole, UK) $10 \mu \mathrm{g} \mathrm{ml}^{-1}$ in PSS; excitation/ emission 495/640-nm) and Hoescht 33342 (Sigma-Aldrich, Poole, UK, 100nmol 1-1 excitation/ emission 350/461-nm). Confocal images were processed using Volocity software (Lexington, MA).

Reactive oxygen species measurement: Intact segments of artery were incubated with $10 \mu \mathrm{mol} \mathrm{l}^{-1}$ 5-(and-6)-chloromethyl-dichlorodihydrofluorescein diacetate, acetyl ester (DCFDA, Invitrogen) for $30 \mathrm{~min}$ and ROS was observed by confocal microscopy in response to stimulation with angiotensin II (ANG II). The vascular smooth muscle layer were measured in the z-axis using the x60 water immersion objective (Nikon N.A. 1.3). A z-plane stack of images were collected where the step interval was set at $1 \mu \mathrm{M}$. The medial smooth muscle layer was determined by the first brightest to last brightest nuclei. The mean fluorescent intensity from each z-stack image was measured and these values averaged to give a mean fluorescent intensity for the whole smooth muscle layer using ImageJ.

\subsection{Drugs and reagents}

Physiological saline solution (PSS): composition (mmol l-1) - $\mathrm{NaCl} 119, \mathrm{KCl} 4.5, \mathrm{NaHCO}_{3}$ 25, $\mathrm{KH}_{2} \mathrm{PO}_{4}$ 1.0, $\mathrm{MgSO}_{4}$ 1.2, glucose 10 and $\mathrm{CaCl}_{2}$ 2.5. $\mathrm{Ca}^{2+}$ free PSS composition was identical to that of PSS other than the removal of $\mathrm{Ca}^{2+}$ and addition of $1 \mathrm{mM}$ EGTA. KrebsHEPES composition (mmol l-1) - $\mathrm{NaCl} 118, \mathrm{KCl} 4.5, \mathrm{MgSO}_{4} 1.2, \mathrm{CaCl}_{2} 2.5, \mathrm{KH}_{2} \mathrm{PO}_{4} 1.2$, $\mathrm{NaHCO}_{3} 25$, HEPES 10, glucose 10. All drugs and reagents were purchased from Sigma (Poole, Dorset, UK) and all were prepared on the day of the experiment. Collagenase PSS:

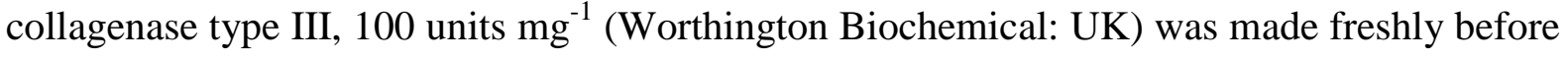
each experiment and diluted with PSS.

\subsection{Data and statistical analysis}

Values are presented as mean \pm standard error of the mean (SEM). Contraction data to vasoconstrictor agonists are represented as percentage contraction relative to the maximum contraction evoked by $60 \mathrm{mmol} \mathrm{l}^{-1} \mathrm{~K}^{+} \mathrm{PSS}$. Relaxation data are represented as percentage relaxation relative to the pre-constricted diameter of the artery. Statistical analysis of the data was performed using Graph Pad Prism (USA). Comparisons between groups were made using paired or unpaired Student's t test or ANOVA for repeated measures. Significance was assumed if $\mathrm{p}<0.05$.

\section{Results}

\subsection{Adventitial removal following collagenase exposure}

Following the 15 minute collagenase $\left(1.5 \mathrm{mg} \mathrm{ml}^{-1}\right)$ exposure protocol (Gonzalez et al 2001) functional integrity was measured. Collagenase exposure alone (with no stripping) under the published experimental conditions resulted in complete loss of fundamental contractile function. Reducing the concentration of collagenase to $0.5 \mathrm{mg} / \mathrm{ml}$ and reducing the time of 
exposure from 15 minutes to 5 minutes failed to improve contractile function (Fig. 1A). Visual analysis of resistance arteries by confocal microscopy revealed propidium iodide positive staining across all layers of the vessels following collagenase exposure (Fig. 1B). In keeping with the original published study collagenase protocols were tested in the larger calibre rat aorta tissue [3]. In aorta collagenase treatment alone or collagenase treatment plus careful stripping of the adventitial layer had no deleterious effect on vascular contractile function nor did it result in any propidium iodide positive staining of the vascular smooth muscle or endothelial layers (data not presented).

\subsection{Adventitial ablation using $4 \%$ formaldehyde buffered saline}

Following 5 second dipping resistance artery function was measured. In contrast to the collagenase exposure protocol FBS exposure had no detrimental effect on receptorindependent contractile function (Fig. 1C). Vascular cellular integrity was confirmed by confocal microscopy. Fig. 1D shows no propidium iodide positive medial or intimal cells following FBS exposure. As expected the outer adventitial layer shows positive staining (Fig. 1D).

\subsection{Functional confirmation of adventitial ablation}

TNS was used to measure neurogenic-dependent contraction. Fig. 2A shows a TNSdependent increase in vascular contractile tone which was significantly reduced following incubation with guanethidine $(10 \mu \mathrm{M})$. The effect of adventitial ablation with transient dipping in 4\% FBS almost eliminated the TNS-dependent response. Additional confirmation of adventitial ablation was investigated using nicotine $(10 \mu \mathrm{M})$ in the presence of guanethidine. Fig. 2B shows significant loss of nicotine-dependent relaxation in ADV-vs. $\mathrm{ADV}+(54.1 \pm 3.1 \%$ vs. $12.8 \pm 3.1 \%$ respectively; $\mathrm{p}<0.05)$. Adventitial fibroblasts have been shown to liberate significant amounts of reactive oxygen species (ROS) [7]. Fig. 2C shows the effect of adventitial ablation on angiotensin II $(0.1 \mathrm{uM})$ induced ROS. Following treatment the capacity of MCAs to liberate ROS as measured by chemiluminescence was significantly reduced to $\sim 50 \%$ in ADV- MCAs (Fig 2C.).

\subsection{Pressure-independent tone}

In MCAs at intraluminal pressure of $40 \mathrm{mmHg}$ and following transient exposure to $4 \% \mathrm{FBS}$ we observed no loss of underlying vascular smooth muscle receptor-independent (Fig. 3A) or receptor-dependent tone (Figs. 3B, 3C). Nor were endothelium-independent (Fig. 3D) or endothelium-dependent relaxation (Fig. 3E) affected.

\subsection{Pressure-dependent tone}

Despite having no effect on pressure-independent mechanism of vascular tone transient dipping in 4\% FBS significantly reduced the well documented pressure-dependent function in MCAs. Fig. $3 \mathrm{G}$ shows pressure-diameter relationship in $\mathrm{ADV}+, \mathrm{ADV}+\mathrm{in}^{2+}$ free $\mathrm{PSS}$ and ADV-. In the presence of $\mathrm{Ca}^{2+}$ and in response to increasing pressure from $40-80-120 \mathrm{mmHg}$ mean vessel diameter reduced (175-165-163 $\mu \mathrm{m}$ respectively). In paraformaldehyde dipped 
vessels loss of pressure-dependent myogenic tone (Fig. 3G) was greatest at $120 \mathrm{mmHg}$ $(\mathrm{ADV}+\mathrm{vs}$. ADV-; $24.8 \pm 2.2 \%$ vs. $9.4 \pm 3.3 \%$ respectively; $\mathrm{p}<0.05)$.

\subsection{Pressure-dependent passive mechanical properties following FBS exposure}

Transient dipping in 4\% FBS may have resulted in partial fixation. Therefore we measured the effect of $4 \%$ formaldehyde exposure on pressure-dependent deformation in an attempt to identify if the loss of pressure-dependent tone in ADV- was due to partial fixation of the outer layer of the artery. However, Fig. 3H highlights there was in fact no degree of partial fixation as measured by the passive pressure-dependent mechanical properties of the arteries. Fig. $3 \mathrm{H}$ exemplifies these observations showing no significant difference in the stress-strain relationship ADV+ vs. ADV-. Moreover no significant difference was observed in the tangent of the elastic modulus values $(\beta)$ in ADV+ vs. ADV-. $(\beta-10.4 \pm 2.4$ vs. $10.1 \pm$ 1.3 respectively).

\subsection{Vascular smooth muscle reactive oxygen species measurement}

Figure 4 shows mean fluorescent activity within the vascular wall in response to angiotensin II $(1 \& 10 \mathrm{uM})$. Of note there is no significant difference between ROS measured in the muscle layer pre and post transient dipping in 4\% FBS. The no loss of function as a consequence of transient dipping in 4\% FBS is further confirmed as we clearly observe similar angiotensin II dose-dependent increase in ROS in both ADV+ and ADV-.

\section{Discussion}

Previously adventitial-dependent functional isolation has been achieved in larger conduit arteries using an enzymatic digest technique [3]. We have confirmed this technique in the present study using rat aorta however we have importantly demonstrated this technique is not suitable for smaller calibre resistance artery studies. In this study we have shown for the first time a technique for isolating adventitial-dependent functional processes in small resistance arteries. We are confident that the method for adventitial ablation only affects adventitial dependent processes. Enzymatic processing resulted in complete loss of tonic mechanisms. The deleterious effect of the enzymatic exposure was quantified by confocal microscopy where the viability of all cells across the vascular wall stained positive for PI. In complete contrast transient dipping in 4\% FBS followed by PSS rinsing produced only PI positive staining of the outer adventitial layer. Functionally the loss of TNS and nicotine dependent tone combined with the functional preservation of the underlying smooth muscle and endothelium function confirms specificity of this method. Additional confirmation of the specificity of functional adventitial ablation using this technique was gained by the loss of ROS as measured by chemiluminescence. Similar to large arteries the adventitial layer of resistance arteries are populated with many fibroblast cells with a large potential to produce ROS [6]. The data presented in Figure $2 \mathrm{C}$ shows a significantly reduced total ROS liberated from the ADV- artery when compared with the ADV+ artery. Whereas figure 4 highlights the amount of ROS measured within the muscle layer of the artery remains unchanged 
suggesting that the reduced ROS measured in the ADV- (Figure 2C) is a consequence of transient 4\% FBS dipping and loss of adventitial function. Thus this technique of isolating adventitial function in small resistance arteries is one potential focus of future research of the role of adventitial fibroblast-derived ROS and vascular reactivity.

One unexpected observation was the effect of adventitial ablation on pressuredependent myogenic tone. To the best of our knowledge this is the first observation showing a potential role of the adventitia layer in modulating acute pressure-dependent myogenic tone. Mechanisms underlying myogenic tone have yet to be fully elucidated. However ROS has been implicated as a co-signalling molecule underlying the myogenic response and adventitial-derived ROS in addition to ROS from the underlying smooth muscle could conceivably be involved in modulating the actin cytoskeleton [8]. Moreover ROS is known to modulate $\mathrm{Ca}^{2+}$ signalling and potassium channel activation $[9,10]$. There is clear lack of consensus regarding the location and nature of the initiating bio-sensor underlying myogenic tone; it is not unconceivable that the location of the sensory apparatus may be situated within the adventitial-medial interface [11]. Clearly this observation requires further investigation however goes some way to exemplifying the potential important application of this technique.

Although the data has not presented within this manuscript similar observations were measured in third order mesenteric arteries thus the implications of this work for resistance artery studies are potentially important. Parallels can be drawn when considering the vascular endothelium in health and disease where the early use of endothelial removal studies to identify endothelial-dependent function were commonly adopted. Following this present study we are now in a position to undertake similar work to further elucidate the role of the adventitial layer in modulating small resistance artery function.

\section{References}

[1] M.J. Mulvany, Modeling the vasculature: a judicious approach? Hypertension 46 (2005) 652-3.

[2] F.A. Auger, P. D'Orléans, L. Germain, Adventitia contribution to vascular contraction: Hints provided by tissue-engineered substitutes Cardiovas Res 75 (2007) 669-678

[3] M.C. González, S.M. Arribas, F. Molero, M.S. Fernández-Alfonso, Effect of removal of adventitia on vascular smooth muscle contraction and relaxation Am J Physiol 280 (2001) 2876-2881.

[4] M.J. Haurani, P.J. Pagano, Adventitial fibroblast reactive oxygen species as autocrine and paracrine mediators of remodeling: Bellwether for vascular disease? Cardiovasc Res 75 (2007) 679-689. 
[5] S. Sartore, A. Chiavegato, E. Faggin, R. Franch, M. Puato, S. Ausoni, Contribution of adventitial fibroblasts to neointima formation and vascular remodeling: from innocent bystander to active participant. Circ Res 89 (2001) 1111-21.

[6] P. Coats, Y.P. Jarajapu, C. Hillier, J.C McGrath, C. Daly, The use of fluorescent nuclear dyes and laser scanning confocal microscopy to study the cellular aspects of arterial remodelling in human subjects with critical limb ischaemia. Exp Physiol 88 (2003) 547-54.

[7] M.J. Mulvany, W Halpern, Contractile properties of small arterial resistance vessels in spontaneously hypertensive and normotensive rats. Circ Res 41 (1977) 19-26.

[8] P.T. Nowicki, S. Flavahan, H. Hassanain, S. Mitra, S. Holland, P.J. GoldschmidtClermont, N.A. Flavahan, Redox signaling of the arteriolar myogenic response. Circ Res 89 (2001) 114-6.

[9] K. Tokube, T. Kiyosue, M. Arita, Effects of hydroxyl radicals on KATP channels in guineapig ventricular myocytes. Pflügers Arch 437 (1998)155-157.

[10] K.M. Lounsbury, Q. Hu, R.C. Ziegelstein, Calcium signaling and oxidant stress in the vasculature. Free Radic Biol Med 28 (2000) 1362-9.

[11] D.R. Harder, J. Narayanan, D. Gebremedhin, Pressure-induced myogenic tone and role of 20-HETE in mediating autoregulation of cerebral blood flow. Am J Physiol 300 (2011) H1557-65.

\section{Figure legends}

Figure 1. The effect of collagenase-dependent adventitial stripping on microvascular function. A. Contractile response to $60 \mathrm{mM} \mathrm{K}{ }^{+} \mathrm{PSS}$ in ADV+ and following 3 min exposure to collagenase $\left(0.2 \mathrm{mg} \mathrm{ml}^{-1}\right)$ collagenase treated and collagenase treated plus adventitial stripping of mid-cerebral resistance arteries. $\mathrm{n}=4$ pairs; ${ }^{*} \mathrm{p}<0.05$, t-test, treatment vs. ADV+. (B) Confocal image of a mid-cerebral resistance artery following $3 \mathrm{~min}$ exposure to collagenase $(0.2 \mathrm{mg} / \mathrm{ml})$ showing propidium iodide positive red staining in all layers of the artery wall (C) Contractile response of mid-cerebral resistance arteries to $60 \mathrm{mmol}^{-1} \mathrm{~K}^{+} \mathrm{PSS}$ before and after $5 \mathrm{sec}$ dipping in $4 \%$ formalin buffered saline ( $\mathrm{n}=9$ pairs) (D) Confocal image of propidium iodide (PI) stained mid-cerebral resistance artery following $5 \mathrm{sec}$ dipping in $4 \%$ formalin buffered saline. Red nuclei are positive for PI; autofluorescing structures within the artery wall shown by the green. (Scale bar $1 \mathrm{~B}$ and $1 \mathrm{D}=50 \mu \mathrm{m}$ ) 
Figure 2. Adventitial-dependent microvascular function following paraformaldehyde exposure. A, TNS-dependent contraction at $16 \mathrm{~Hz}$ before and after incubation with guanethidine $(10 \mu \mathrm{M})$ in $\mathrm{ADV}+$ arteries and $16 \mathrm{~Hz}$-dependent contraction in ADV-arteries; Contraction data are expressed as a $\%$ of the maximum contraction to $60 \mathrm{mmol}^{-1} \mathrm{~K}^{+} \mathrm{PSS}$. * $\mathrm{p}<0.05$ ADV+ vs. Guanethidine/ ADV+, n=6, t-test; \# p<0.05 ADV-vs. ADV+, n=6, t-test. $\mathrm{B}$, Shows nicotine-dependent relaxation (in the presence of $10 \mu \mathrm{M}$ guanethidine); $* \mathrm{p}<0.05, \mathrm{t}$ test, $\mathrm{ADV}$ - vs. $\mathrm{ADV}+, \mathrm{n}=6 . \mathrm{C}$, Measurement of reactive oxygen species by chemiluminescence in angiotensin II $(0.1 \mu \mathrm{M})$ challenged MCAs. * $\mathrm{p}<0.05, \mathrm{ADV}-\mathrm{vs}$. ADV+, one way ANOVA for repeated measures, $n=6$.

Figure 3. Highlights contractile functional analysis in resistance arteries following transient dipping in 4\% FBS. A, receptor-independent mechanisms of contraction evoked by potassium supplemented PSS (4.5-100 $\left.\mathrm{mmol}^{-1} ; \mathrm{n}=6\right)$. B and C highlight receptordependent contraction evoked by U46199 (n=6) and 5-hydroxytryptamine (5HT; n=6) respectively. $\mathrm{D}$ and $\mathrm{E}$ show the effect on endothelium-independent relaxation and endothelium-dependent relaxation evoked by sodium nitroprusside (SNP; n=6) and acetylcholine (ACh; $n=6$ ) respectively. $F$, pressure-diameter relationship in rat mid-cerebral resistance arteries. * $\mathrm{p}<0.05 \mathrm{ADV}$ - vs. $\mathrm{ADV}+(\mathrm{ANOVA}) \mathrm{n}=9$, and pressure-diameter relationship following removal of $\mathrm{Ca}^{2+} \# \mathrm{p}<0.05 \mathrm{ADV}+/ \mathrm{ADV}$ - vs. $\mathrm{Ca}^{2+}$ free $(\mathrm{n}=7)$. G, effect of paraformaldehyde dipping on pressure-dependent myogenic tone, * $\mathrm{p}<0.05$ ADV- vs. $\mathrm{ADV}+$ at 80 and $120 \mathrm{mmHg}$, ANOVA for repeated measures, $\mathrm{n}=9$. $\mathrm{H}$, highlights no change in pressure-dependent mechanical properties as measured by the stress-strain relationship in $\mathrm{ADV}+$ and $\mathrm{ADV}$ - arteries, $\mathrm{n}=8$.

Figure 4. Measurement of ROS in the vascular smooth muscle wall of small resistance arteries using laser scanning confocal microscopy. Stimulation with $1 \mathrm{uM}$ angiotensin II (Ang II) produced $\sim 3.5$ fold increase in ROS when compared with background unstimulated control $(\bullet p<0.05,1 \mathrm{uM}$ Ang II vs. Control, $\mathrm{n}=6)$. The liberation of ROS further increased to $\sim 6$ fold above background when stimulated with $10 \mathrm{uM}$ Ang II and was again significantly increased when compared with 1uM Ang II (* $\mathrm{p}<0.0510 \mathrm{uM}$ Ang II vs. 1uM Ang II, $n=6)$. In all cases there were no differences measured when comparing ADV- vs. ADV+. 